\title{
OPEN Insights into intrauterine growth restriction based on maternal and umbilical cord blood metabolomics
}

\author{
Georgios Moros ${ }^{1,2,6}$, Theodora Boutsikou 3,6 , Charalambos Fotakis ${ }^{2}$, Zoe Iliodromiti ${ }^{3}$, \\ Rozeta Sokou ${ }^{3}$, Theodora Katsila ${ }^{2}$, Theodoros Xanthos ${ }^{4}$, Nicoletta lacovidou ${ }^{3 凶}$ \& \\ Panagiotis Zoumpoulakis ${ }^{2,5}$
}

Intrauterine growth restriction (IUGR) is a fetal adverse condition, ascribed by limited oxygen and nutrient supply from the mother to the fetus. Management of IUGR is an ongoing challenge because of its connection with increased fetal mortality, preterm delivery and postnatal pathologies. Untargeted nuclear magnetic resonance $\left({ }^{1} \mathrm{H}\right.$ NMR) metabolomics was applied in 84 umbilical cord blood and maternal blood samples obtained from 48 IUGR and 36 appropriate for gestational age (AGA) deliveries. Orthogonal projections to latent structures discriminant analysis (OPLS-DA) followed by pathway and enrichment analysis generated classification models and revealed significant metabolites that were associated with altered pathways. A clear association between maternal and cord blood altered metabolomic profile was evidenced in IUGR pregnancies. Increased levels of the amino acids alanine, leucine, valine, isoleucine and phenylalanine were prominent in IUGR pregnancies indicating a connection with impaired amino acid metabolism and transplacental flux. Tryptophan was individually connected with cord blood discrimination while 3-hydroxybutyrate assisted only maternal blood discrimination. Lower glycerol levels in IUGR samples ascribed to imbalance between gluconeogenesis and glycolysis pathways, suggesting poor glycolysis. The elevated levels of branched chain amino acids (leucine, isoleucine and valine) in intrauterine growth restricted pregnancies were linked with increased insulin resistance.

An emerging body of literature indicates that abnormal fetal growth is associated with increased risk of perinatal morbidity and mortality ${ }^{1-3}$. More specifically, intrauterine growth restriction (IUGR) is associated with chronic hypoxia and stress, leading to adverse endocrine axis reprogramming ${ }^{4,5}$. The aforementioned alterations predispose IUGR infants to impaired glucose homeostasis, insulin sensitivity and adipose tissue development, factors that are associated with coronary vascular disease, hypertension and diabetes mellitus in adulthood ${ }^{6,7}$.

IUGR results from the failure of the fetus to reach its intrinsic growth potential, due to maternal, fetal, or placental pathology ${ }^{8}$. The main cause of IUGR is placental insufficiency ${ }^{9}$, causing impaired transfer of nutrients to the fetus due to limited blood flow ${ }^{10}$.

The incidence of IUGR is approximately $8 \%$ in total population ${ }^{11}$. Different rates have been recorded in the literature depending on fetal intrinsic factors, as well as prenatal follow-up and optimal perinatal care ${ }^{12}$.

Considering that fetal growth along with gestational age at birth determine neonatal survival, as well as short and long-term complications ${ }^{1,13}$, it is critical to elucidate the underlying pathogenesis of IUGR and the respective implicated metabolic pathways.

Prenatal diagnosis of IUGR mainly relies on abnormal umbilical and uterine Doppler measurements that enable real time estimation of placental/fetal circulation, but do not permit early intervention. Diagnosis is also assisted by an ultrasound-estimated fetal weight below the corresponding 10th percentile for the gestational week $^{14}$.

\footnotetext{
${ }^{1}$ Department of Biochemistry \& Biotechnology, University of Thessaly, Larisa, Greece. ${ }^{2}$ Institute of Chemical Biology, National Hellenic Research Foundation, Athens, Greece. ${ }^{3}$ Department of Neonatology, National and Kapodistrian University of Athens, Aretaieio Hospital, Athens, Greece. ${ }^{4}$ School of Medicine, European University, Nicosia, Cyprus. ${ }^{5}$ Department of Food Science and Technology, University of West Attica, Athens, Greece. ${ }^{6}$ These authors contributed equally: Georgios Moros and Theodora Boutsikou. ${ }^{\varpi}$ email: niakobid@med.voa.gr; pzoump@uniwa.gr
} 

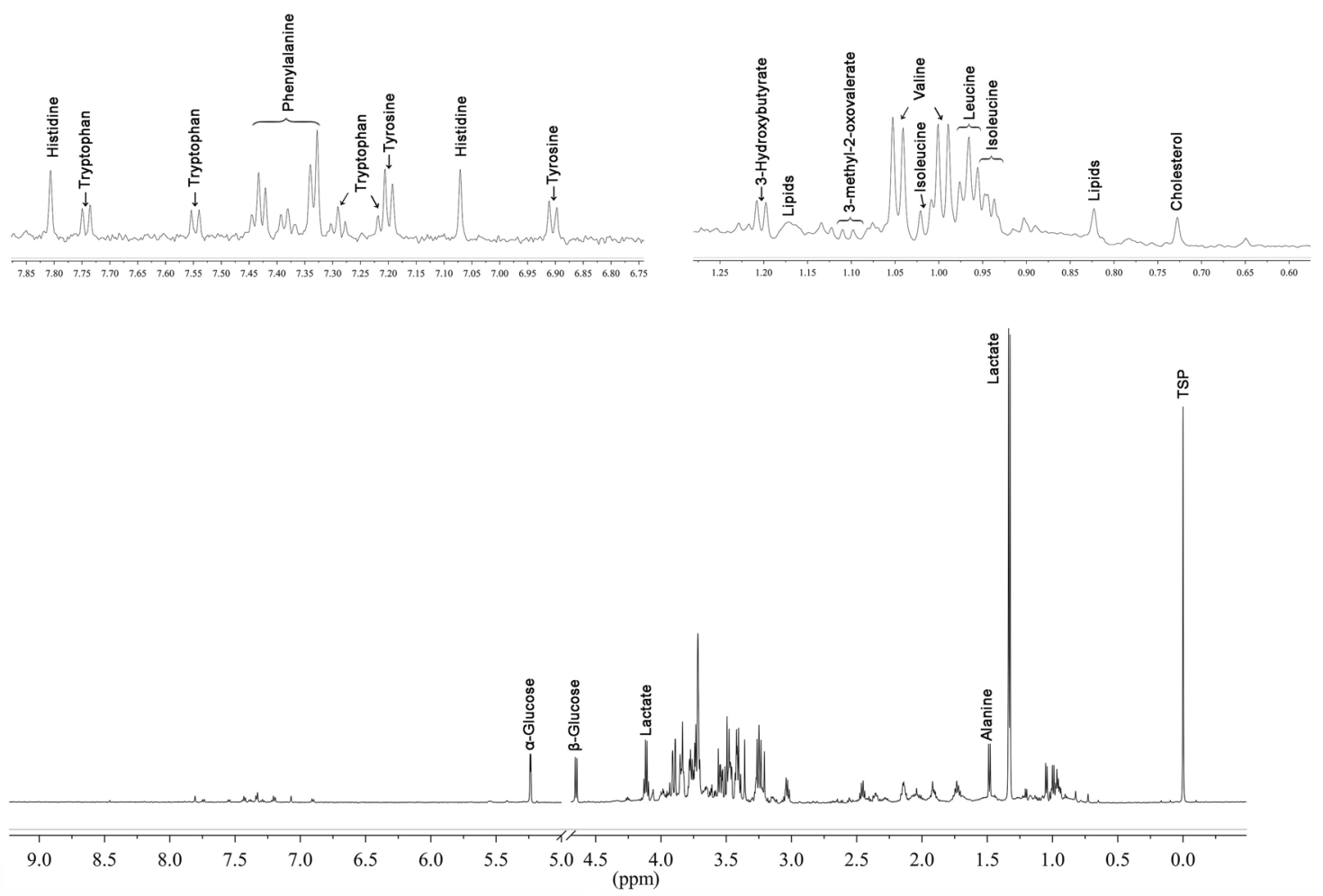

Figure 1. Typical ${ }^{1} \mathrm{H}-\mathrm{NMR}$ spectrum of serum after methanol extraction following cpmgpr pulse sequence with expanded regions; the region between 3.25 and $4.00 \mathrm{ppm}$ is occupied by sugar resonances. Major metabolites are assigned over the spectrum peaks.

Thus, it is essential to determine biomarkers framing the time of insult or maternal-fetal metabolic interaction suggesting early intervention. Although several metabolites have been suggested in literature as putative biomarkers for IUGR screening, still none is generally applied in clinical practice ${ }^{15-18}$.

The current study presents a nuclear magnetic resonance (NMR) based untargeted metabolomic approach focusing on IUGR pathology with a twofold aim; (a) to provide explanatory models for IUGR pathogenesis and (b) to identify putative markers of prediction which can be validated at the early stages of pregnancy. Both umbilical cord and maternal blood from well-defined IUGR and appropriate for gestational age (AGA) cases were investigated in one of the biggest sample sizes compared to relevant studies.

\section{Results}

Assignment for 56 individual metabolites excluding the lipid and sugar regions is presented in Supplementary Table S1. Typical ${ }^{1} \mathrm{H}-\mathrm{NMR}$ spectra with characteristic annotations is presented in Fig. 1.

Exploratory analysis. Principal component analysis (PCA) on NMR data was implemented in each of the four studied groups (IUGR cord and maternal samples, AGA cord and maternal samples) to assess sample homogeneity and pinpoint outliers. Several characteristics including maternal pathology, smoking habits, gestational age, maternal age, maternal BMI, mode of delivery and gender were examined in each of the produced statistical models. Maternal pathology testing is shown in Supplementary Figs. S1-S4.

Six IUGR participants with gestational diabetes mellitus who presented increased glucose levels and one participant with thrombophilia with a distinct profile were excluded from further investigation from the IUGR group (maternal and cord samples). Furthermore, in umbilical cord blood samples (41 IUGR and 36 AGA), a discrimination trend was observed among IUGR and AGA samples based on the mode of delivery (Supplementary Fig. S5). Samples from vaginal deliveries presented higher lactate levels compared to those from cesarean sections, which necessitates the exclusion of their signals [1.33 (d) and $4.11(\mathrm{q})$ ] from further data processing. After removing the lactate resonances the impact of labor on blood metabolites was effaced, since no grouping was observed based on the delivery mode.

Investigation of IUGR-AGA umbilical cord blood samples. A general overview of the remaining cord blood samples (41 IUGR and 36 AGA) after the removal of lactate and methanol regions is presented in Fig. 2. AGA samples (blue circles) tend to cluster in the first quadrant, while IUGR samples (red circles) are more concentrated in the second, third and fourth quadrants. Figure 3a,b illustrate the OPLS-DA scores and loadings plot for the studied pair, respectively. Permutation testing with receiver operator characteristic (ROC) curves validated the OPLS-DA discrimination (Supplementary Fig. S6a). 


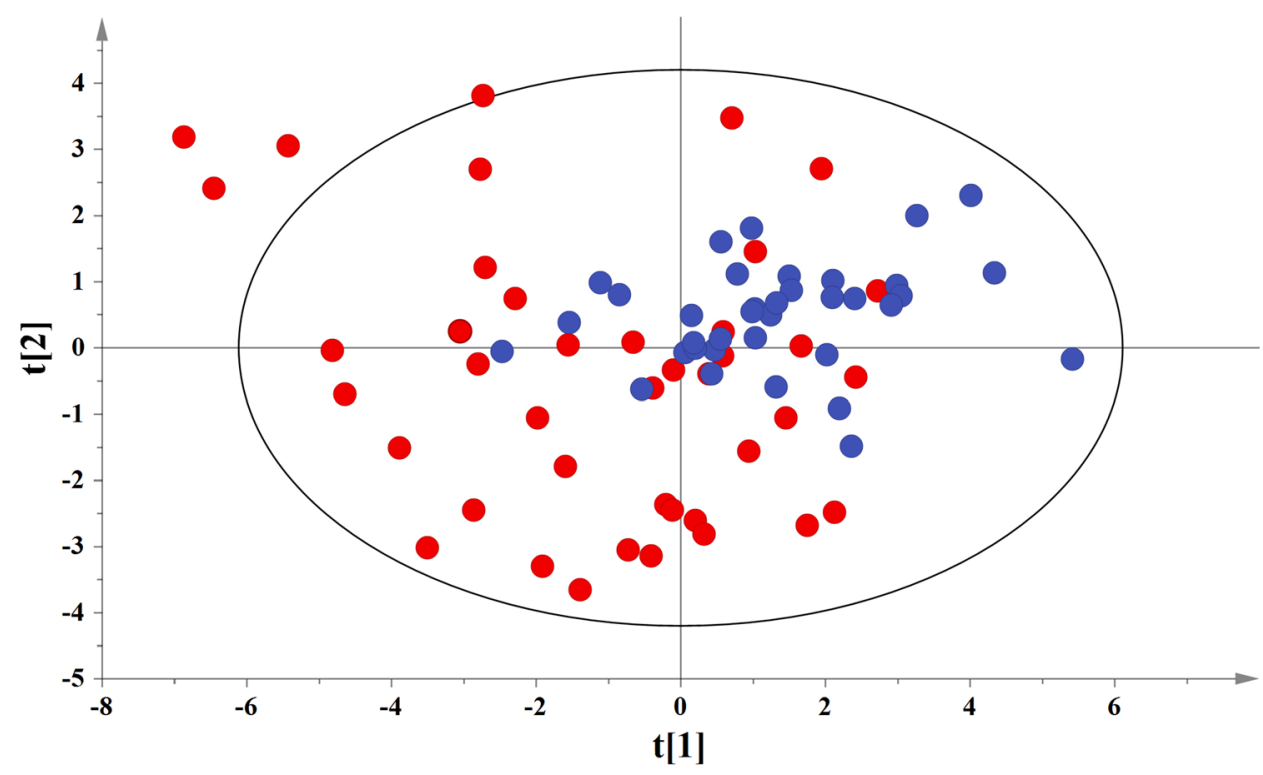

Figure 2. PCA scores plot among the umbilical cord blood samples set. IUGR and AGA samples are represented with red and blue circles, respectively. $A=8, N=77, R^{2} X(\mathrm{cum})=0.642, Q^{2}(\mathrm{cum})=0.451$ for pareto scaling and 95\% confidence level. $\mathrm{t}[1]$ Eigenvalue:16.6, $\mathrm{t}[2]$ Eigenvalue:7.77.

As depicted in Fig. 3b, IUGR umbilical cord blood samples are characterized by higher levels of the branched chain amino acids (BCAAs) leucine, isoleucine and valine and the non-essential amino acid alanine. On the other hand, AGA samples are characterized by higher levels of the essential aminoacids phenylalanine and tryptophan. Moreover, glycerol is altered between the two test-groups, being elevated in AGA samples. Biomarker analysis (Supplementary Fig. S7) confirmed the above findings for the suggested metabolites between the two groups revealing a high Area Under the Receiver Operating Characteristic curve (AUROC).

Investigation of IUGR-AGA maternal blood samples. A similar data analysis process was followed among maternal samples. Figure 4 illustrates a trend of clustering among the second principle component of the PCA. More specifically IUGR samples (red circles) create a cluster among the 3 rd and 4th quadrants while AGA samples (blue circles) among the 1st and 2nd quadrants. Figure 5a,b illustrate the OPLS-DA scores and loadings plot for the studied pair, respectively. Validation tests are presented in Supplementary Fig. S6b.

The metabolites assigned to the discriminatory bins of the loadings plot (Fig. 5b) along with significant AUROC (Supplementary Fig. S8), correspond to the essential amino acids leucine, isoleucine, valine and phenylalanine, the non-essential amino acid alanine and the organic metabolites namely glycerol and 3-hydroxybutyrate.

Significant metabolites and altered pathways. Table 1 presents the AUROCs for the discriminatory metabolites between test-groups. Univariate testing is presented in Table 2.

Pearson correlation testing between the peak integral areas of the remaining lipids at 0.82 and $1.16 \mathrm{ppm}$ with the BCAAs and the 3-hydroxybutyrate areas for 10 representative samples of each group (umbilical and maternal) probed to no significant correlation (weak to moderate values of the correlation coefficient) (Supplementary Tables S2 and S3). Interestingly, 6 out of 8 proposed metabolites were prominent in both umbilical cord blood and maternal group discrimination with high degree of statistical significance (AUROC $>0.75$ and $p$-value $<0.05$ ). In particular, higher levels were recorded for the branched chain amino acids BCAAs (leucine, isoleucine and valine) and alanine in IUGR groups, in contrast to the increased phenylalanine and glycerol levels in the AGA groups.

On the other hand, tryptophan contributed only to the umbilical cord blood discrimination, whereas 3-hydroxybutyrate only to the maternal blood discrimination. Tryptophan showed increased levels in AGA umbilical cord blood samples and 3-hydroxybutyrate showed increased levels in IUGR maternal blood samples. Alanine is the most significant metabolite $($ AUROC $=0.871)$ for cord blood discrimination and isoleucine (AUROC $=0.812$ ) for maternal discrimination.

Enrichment analysis provided the deregulated pathways due to the IUGR pathology. Supplementary Tables S4 and S5 present the three most probably affected pathways in umbilical cord blood and maternal blood sample pairs respectively. Pathways with the higher number of hits and a p value lower than 0.05 (statistically significant) were selected as the candidate key pathways. The most significantly affected metabolic pathway in both pairs reflects the Valine, Leucine and Isoleucine Degradation. 


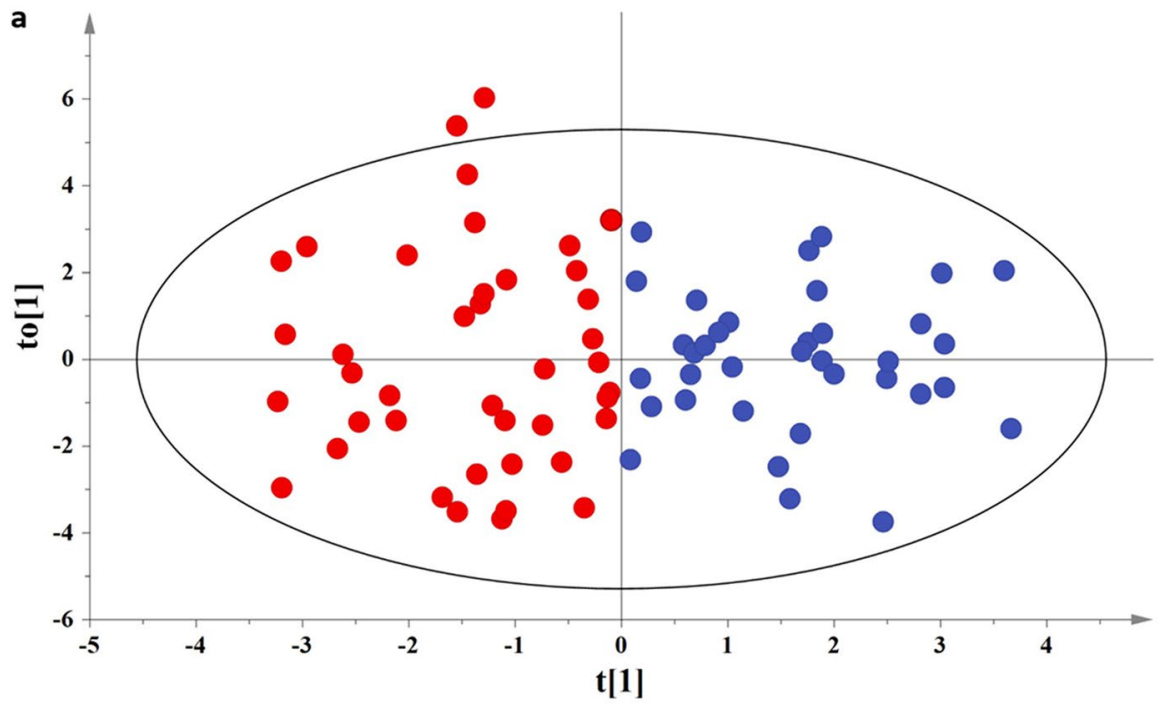

b

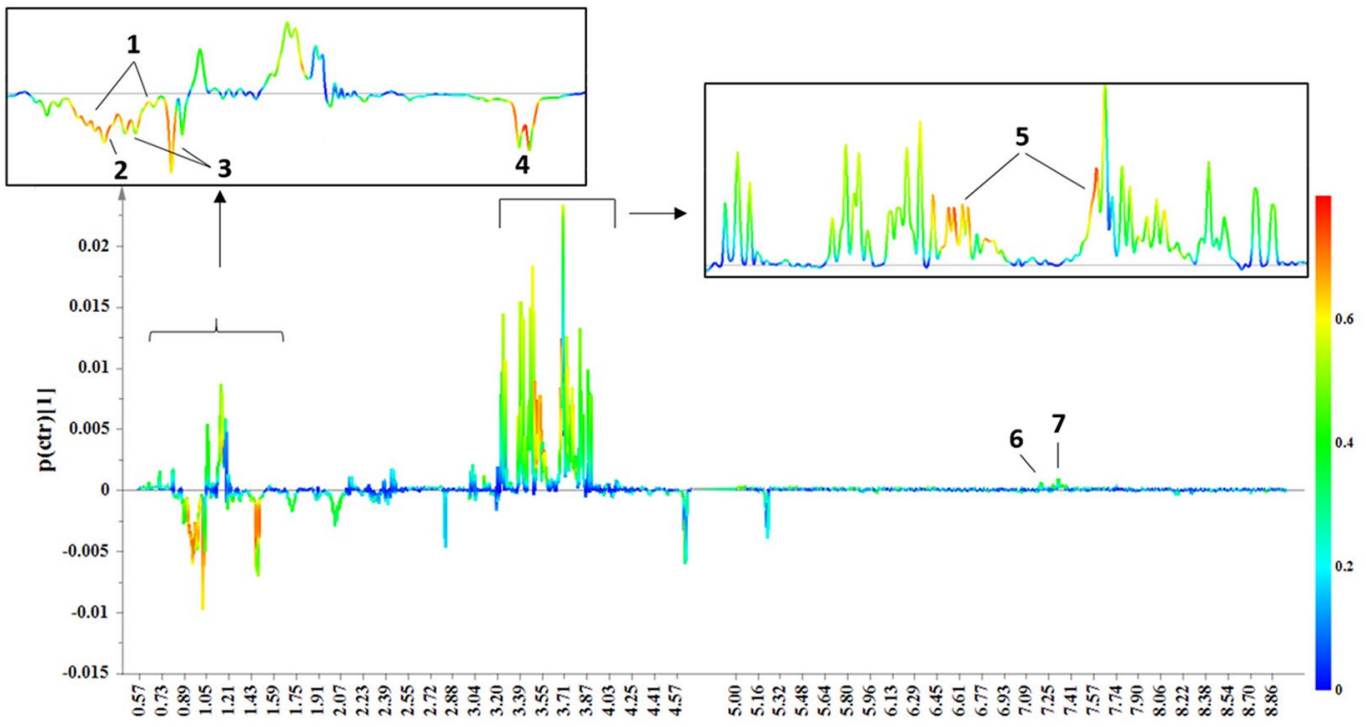

Figure 3. (a) OPLS-DA scores for the umbilical cord blood samples, with $A=1+1+0, N=77$, $\mathrm{R}^{2} \mathrm{X}(\mathrm{cum})=0.279, \mathrm{R}^{2} \mathrm{Y}(\mathrm{cum})=0.70, \mathrm{Q}^{2}$ (cum) $=0.559$ for pareto scaling and $95 \%$ confidence level. Red circles denote IUGR and blue circles AGA samples. (b) Loadings plot demonstrate metabolites responsible for discrimination, 1: Isoleucine, 2: Leucine, 3: Valine, 4: Alanine, 5: Glycerol, 6: Tryptophan, 7: Phenylalanine.

\section{Discussion}

A similar metabolic pattern was evident for the first time between maternal and umbilical cord blood in IUGR pregnancies. This finding may indicate common mechanisms underlying the IUGR condition which connected between mothers and fetuses and can pave the way for the identification of putative biomarkers in maternal blood targeting early IUGR screening.

Controversial information is evidenced in the literature concerning candidate metabolites for IUGR-AGA discrimination, with abnormal amino acid metabolism, glucose intolerance and limited insulin resistance being an important finding ${ }^{19}$.

IUGR off springs have altered protein expression profile ${ }^{20}$ which may result in a reduced utilization rate of the BCAAs and alanine, leading to a consequent increase of their levels. This increase could also be explained by the observation that IUGR fetuses experience the same metabolic derangements with certain diseases, with urea cycle defects being the most relevant ${ }^{21}$.

Starving conditions promote protein degradation leading to the consequent increase of BCAAs. These are degraded in tissues, other than liver, yielding reduced $\mathrm{NADH}$ and $\mathrm{FADH}_{2}$ which facilitate the energy production $^{22}$. IUGR is linked to reduced oxygen and nutrients supply through the placenta ${ }^{23}$ which could partly explain the observed BCAAs increase. Nutrient deficiency has also been proposed to describe the up-regulation of valine and isoleucine in the IUGR vs AGA groups ${ }^{16}$. 


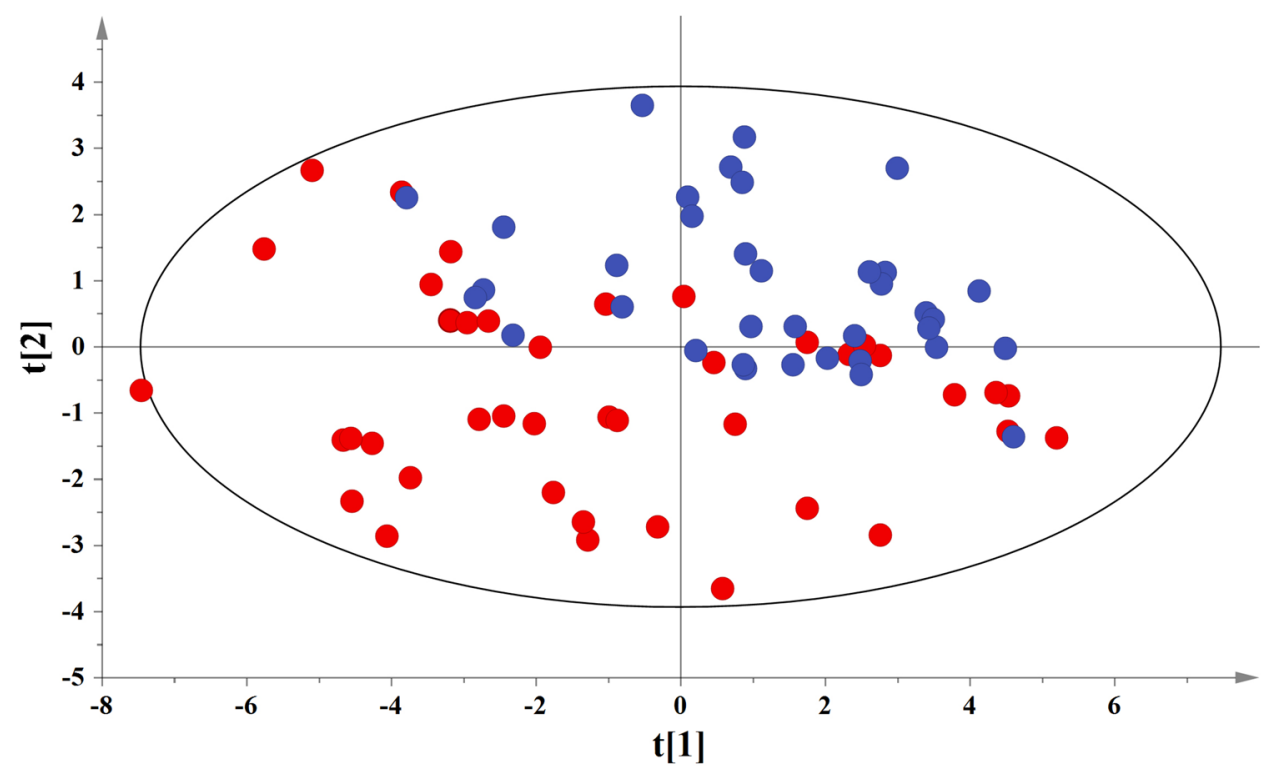

Figure 4. PCA scores plot among the maternal blood samples set. IUGR and AGA samples are represented with red and blue circles, respectively. $A=8, N=77, R^{2} X($ cum $)=0.666, Q^{2}$ (cum) $=0.506$ for pareto scaling and 95\% confidence level. t[1] Eigenvalue:22.7, t[2] Eigenvalue:6.3.

Moreover, increased levels of BCAAs have been associated with insulin resistance and future type 2 diabetes ${ }^{24-26}$. Dessi et al. ${ }^{18}$ suggested that IUGR fetuses stimulate a number of adaptive mechanisms in order to save glucose and promote nutrition of the vital organs, resulting in down-regulation of insulin secretion. Herein, the observed increase of BCAAs in the IUGR group could be associated with insulin resistance of the fetus. Glucose intolerance or limited insulin adequacy in IUGR, may account for altered tricarboxylic acid (TCA) metabolic intermediates, such as alanine and leucine ${ }^{27}$.

In a previous type 2 diabetes metabolomic study ${ }^{26}$, the branched-chain keto acid (BCKA) 3-methyl-2-oxovalerate, has been identified as one of the strongest predictors of impaired fasting glucose (IFG). This BCKA arises from the incomplete catabolism of BCAAs ${ }^{26,28}$ which primarily occurs in the mitochondria. High levels of the corresponding metabolite are also linked with the metabolic disorder of maple syrup urine disease that is caused by the deficiency of the branched-chain alpha-keto acid dehydrogenase complex, leading to the accumulation of BCAAs ${ }^{28}$.

The glucose-alanine cycle describes the synthesis of glucose from alanine in the liver and the transport of glucose back to the muscles. Alanine serves as a precursor or regulator of glucose metabolism that is of critical importance in the nutrient-deprived IUGR, thus explaining the increased alanine levels found in the IUGR group $^{22}$. Higher alanine levels were also found for IUGR having a birth weight between 3rd and 10th percentile ${ }^{29}$.

In our research, lower glycerol levels were observed in the IUGR group, signifying glycerol deprivation. Gluconeogenesis is a pathway describing glucose synthesis from lactate, pyruvate, glycerol and glucogenic amino $\operatorname{acids}^{30}$. In the liver, glycerol may be converted into glucose, meeting the required energy needs for cellular metabolism ${ }^{31}$. Glycolysis, a compensatory pathway that breakdowns glucose, is deregulated in conditions of limited insulin adequacy or resistance ${ }^{32}$. IUGR infants have insulin deficiency ${ }^{18}$ which partly explains poor glycolysis together with a continuous conversion of glycerol to glucose through gluconeogenesis. Also, lower glycerol levels in the IUGR groups could indicate an imbalance between triacylglycerol breakdown and re-synthesis during several metabolic reactions in a continuous flux between adipose tissue and liver.

In pregnant women with glucose intolerance, ketone body formation is favored against carbohydrate consumption $^{33}$, resulting in the accumulation of 3-hydroxybutyrate. Furthermore, the observed increase of the ketogenic essential amino acids leucine and isoleucine, may also account for increased 3-hydroxybutyrate synthesis $^{33}$. The metabolomic study of Powel et al. ${ }^{34}$ suggested 3-hydroxybutyrate is a predictor of IUGR complication in maternal serum as well, however, with low predictive power.

Different brain serotonin synthesis has been associated with altered tryptophan levels ${ }^{16}$. Cosmi et al. ${ }^{35}$ in line with our observations indicated tryptophan as a candidate marker of IUGR pathology, but reported findings are different to our results regarding our observed up-regulation of valine and isoleucine and the down-regulation of phenylalanine. Of note, such outcomes refer to twin neonates and are deprived by limited sampling.

Paolini et al. ${ }^{36}$ found a lower fetal/maternal enrichment ratio for phenylalanine and leucine in IUGR compared to normal pregnancies, suggesting an impaired transplacental flux. An altered placental metabolism or transport alongside with a higher catabolic condition has been also suggested to explain the lower phenylalanine levels in the IUGR samples ${ }^{16,37}$. The fetomaternal ratio for the essential amino acids is reduced in cases of small for gestational age (SGA) compared to AGA, as a result of fetal hypoxemia ${ }^{38}$. Finally, increased levels of BCAAs 
a

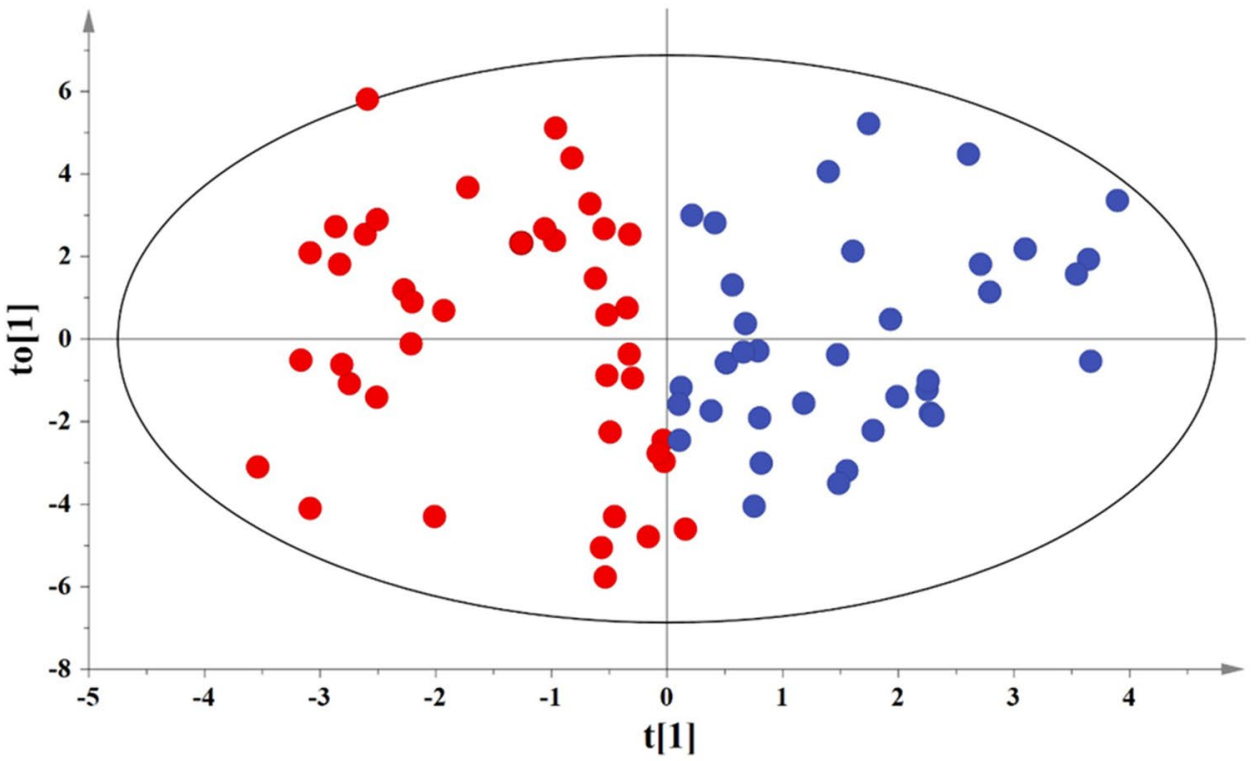

b

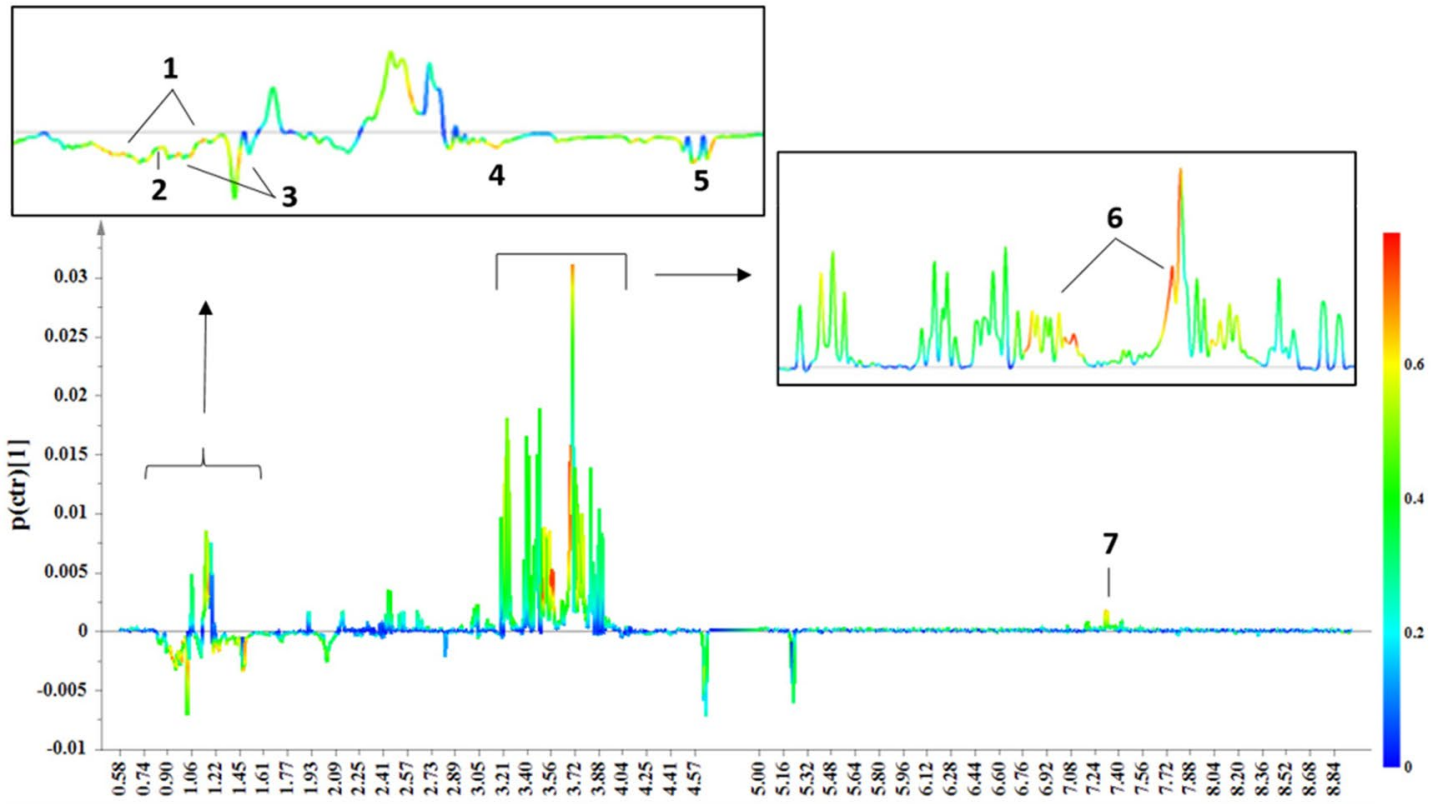

Figure 5. (a) OPLS-DA scores plot for maternal samples with $A=1+1+0, N=77, R^{2} X($ cum $)=0.369$, $\mathrm{R}^{2} \mathrm{Y}(\mathrm{cum})=0.65, \mathrm{Q}^{2}(\mathrm{cum})=0.566$ for pareto scaling and $95 \%$ confidence level. IUGR are represented with red circles and AGA correspond to blue circles. (b) Loadings plot demonstrate the metabolites responsible for discrimination, 1: Isoleucine, 2: Leucine, 3: Valine, 4: 3-Hydroxybutyrate, 5: Alanine, 6: Glycerol, 7: Phenylalanine.

and alanine in the maternal blood of IUGR vs AGA groups, is possibly explained due to the altered adaptation mechanisms of IUGR pregnancy with incomplete hormone production ${ }^{38,39}$.

\section{Conclusions}

In our study we interpreted the generated metabolomic data and attempted to correlate a fetal adverse condition with alterations in the metabolic profile as putative biomarkers. A similar metabolic profile was evident between maternal and umbilical cord blood in IUGR pregnancies, with seven out of 56 identified metabolites being responsible for discrimination. As an exception, tryptophan contributed only to cord blood discrimination while 3-hydroxybutyrate only to maternal blood discrimination.

Higher levels of the BCAAs, together with alanine were observed in the IUGR groups, in contrast to increased phenylalanine and glycerol levels for the AGA groups, indicating the crucial roles of amino acid metabolism, insulin resistance and glycolysis pathway. The elevated levels of BCAAs in intrauterine growth restricted pregnancies 


\begin{tabular}{|l|l|l|}
\hline \multirow{2}{*}{ Altered metabolite } & AUROC \\
\cline { 2 - 3 } & Umbilical cord blood & Maternal blood \\
\hline Alanine & $\mathbf{0 . 8 7 1}$ & 0.792 \\
\hline Leucine & 0.816 & 0.773 \\
\hline Isoleucine & 0.795 & $\mathbf{0 . 8 1 2}$ \\
\hline Valine & 0.785 & 0.786 \\
\hline Phenylalanine & 0.779 & 0.75 \\
\hline Glycerol & 0.835 & 0.751 \\
\hline Tryptophan & 0.751 & Non-significant \\
\hline 3-Hydroxybutyrate & Non-significant & 0.774 \\
\hline
\end{tabular}

Table 1. Comparison of AUROCs for discriminatory metabolites among umbilical cord blood and maternal samples. Values in bold refer to metabolites presenting the higher AUROC.

\begin{tabular}{|l|l|l|l|l|}
\hline \multirow{2}{*}{ Altered metabolite } & \multicolumn{2}{|l|}{ Umbilical cord blood } & Maternal blood \\
\cline { 2 - 5 } & $\mathbf{p}$-value & Fold changes ${ }^{\mathbf{a}}$ & p-value & \multicolumn{1}{|l|}{ Fold changes $^{\mathbf{a}}$} \\
\hline Alanine & $6.47 \times 10^{-9}$ & -0.47 & $3.76 \times 10^{-6}$ & -0.54 \\
\hline Leucine & $1.31 \times 10^{-6}$ & -0.42 & $5.81 \times 10^{-5}$ & -0.32 \\
\hline Isoleucine & $6.39 \times 10^{-6}$ & -0.44 & $8.76 \times 10^{-7}$ & -1.07 \\
\hline Valine & $4.77 \times 10^{-6}$ & -0.47 & $7.18 \times 10^{-6}$ & -0.48 \\
\hline Phenylalanine & $3.05 \times 10^{-5}$ & 2.42 & $9.73 \times 10^{-5}$ & 0.41 \\
\hline Glycerol & $1.59 \times 10^{-7}$ & 0.45 & $1.84 \times 10^{-4}$ & 0.26 \\
\hline Tryptophan & $7.52 \times 10^{-5}$ & 3.17 & Not-significant & Not-significant \\
\hline 3-Hydroxybutyrate & Non-significant & Non-significant & $2.58 \times 10^{-5}$ & -0.50 \\
\hline
\end{tabular}

Table 2. P-values and fold changes for significant metabolites among umbilical and maternal blood samples. ${ }^{\text {a }}$ Positive values indicate metabolites determined in higher concentration in the AGA group whereas negative values indicate metabolites determined in higher concentration in the IUGR group.

were linked with increased insulin resistance. Although a number of clinical studies have facilitated metabolomics to elaborate on the IUGR condition, biomarkers that determine this condition early on, are still lacking. From our findings, the role of previously reported metabolites has been strengthened together with the significance of 3-hydroxybutyrate in IUGR maternal blood. The proposed metabolic signatures have to be validated at an earlier stage of pregnancy in order to obtain clinical impact as diagnostic and prognostic biomarkers for IUGR.

\section{Methods}

Ethical statement. An approval (No. EE-2/26/06-06-2017) from the Aretaieio Hospital Review Board along with the Ethics committee was received before the experiments were started. All experiments were performed in accordance relevant guidelines and regulations. Signed informed consent was obtained from the participating mothers prior to enrollment.

Patient recruitment. The study comprised 84 parturients giving consecutively birth to 48 IUGR ( $\leq 10$ th customized centile) and 36 AGA (between 10 and 90th customized centile) full-term infants (Supplementary Table S6). Among IUGR pregnancies, 6 were complicated by gestational diabetes mellitus (GDM) and 1 by thrombophilia. Median gestational age for IUGR neonates was 273 ( \pm 7 days) and median birth weight was 2610 ( $\pm 263 \mathrm{~g}$ ). Median gestational age for AGA neonates was 278 ( \pm 6 days) and median birth weight was 3405 $( \pm 273 \mathrm{~g})$. Diagnostic testing is presented in Supplementary Methods.

Sample collection and pretreatment. Mixed arteriovenous blood was collected from the doubly clamped umbilical cord, reflecting fetal blood. Maternal blood was collected either at the first stage of labor in cases of vaginal delivery or before receiving anesthesia in cases of caesarean section, in fasting conditions; time between last food intake and sampling were at least $8 \mathrm{~h}$. Samples allowed to clot at room temperature for $1 \mathrm{~h}$ and centrifuged at $1500 \times \mathrm{g}$ for $10 \mathrm{~min}$. Serum was collected in eppendorf tubes and transferred to $-80^{\circ} \mathrm{C}$ till sample preparation.

Serum samples were thawed at room temperature and extracted according to Nagana Gowda et al. ${ }^{40}$ protocol. In particular, $250 \mu \mathrm{L}$ serum samples were extracted with $500 \mu \mathrm{L}$ methanol $(1: 2 \mathrm{v} / \mathrm{v})$, vortexed and placed at $-20^{\circ} \mathrm{C}$ for $20 \mathrm{~min}$. Samples were centrifuged $\left(11,000 \mathrm{rpm} / 4^{\circ} \mathrm{C}\right)$ for $20 \mathrm{~min}$ to allow protein parts to precipitate. Supernatants placed to new eppendorf tubes and evaporated to dryness. Samples were reconstituted to $400 \mu \mathrm{L}$ $\mathrm{D}_{2} \mathrm{O}$ and $150 \mu \mathrm{L}$ phosphate buffer $\left(0.2 \mathrm{M}, \mathrm{Na}_{2} \mathrm{HPO}_{4} 2 \mathrm{H}_{2} \mathrm{O}\right.$ and $\left.\mathrm{NaH}_{2} \mathrm{PO}_{4}, \mathrm{pH}=7.0\right)$. Then, $500 \mu \mathrm{L}$ of each mixture with $50 \mu \mathrm{L}$ of internal standard trimethylsilyl propionic acid sodium salt (TSP) $(2.75 \mathrm{mM})$ were transferred to $5 \mathrm{~mm}$ NMR tubes for ${ }^{1} \mathrm{H}-\mathrm{NMR}$ analysis. 
${ }^{1} \mathrm{H}-\mathrm{NMR}$ spectroscopy. All ${ }^{1} \mathrm{H}-\mathrm{NMR}$ spectra were acquired using a Varian $600 \mathrm{MHz}$ spectrometer equipped with a triple resonance probe $\left({ }^{1} \mathrm{H},{ }^{13} \mathrm{C},{ }^{15} \mathrm{~N}\right)$, at room temperature $\left(25^{\circ} \mathrm{C}\right)$. The CPMG pulse sequence following water suppression was applied in all experiments since it provided better spectra in terms of $\mathrm{S} / \mathrm{N}$ ratio compared to $1 \mathrm{DNOE}$ pulse sequence. In total, 128 transients were collected with $64 \mathrm{~K}$ data points and a relaxation delay of $5 \mathrm{~s}$. Receiver gain was auto set 60 for all acquisitions. All ${ }^{1} \mathrm{H}$ NMR spectra were referenced at TSP chemical shift $(0.00 \mathrm{ppm})$ and processed at 0.3 exponential line broadening.

Data handling and metabolite assignment. All ${ }^{1} \mathrm{H}-\mathrm{NMR}$ spectra were preprocessed with MestreNova (v. 10.1) software. Manual phase correction, automatic baseline correction and sinc apodization were applied to improve spectra resolution. Total area normalization and binning of $0.001 \mathrm{ppm}$ were selected. Superimposed spectrum was constructed and peaks were manually aligned. The water region (4.68-5.00 ppm) was excluded together with the peak at $3.36 \mathrm{ppm}$ attributed to methanol from protein precipitation procedure.

Peak assignment and metabolite identification were performed using 2D NMR experiments (gCOSY, zTOCSY, gHSQCad and gHMBCad) on a representative pool sample. Assignment of spectral peaks was assisted by Chenomx database (Chenomx Suite 7.6, Chenomx, Edmonton, Alberta, Canada), an in-house software (Metaboneer $)^{41}$ and the online NMR databases $\mathrm{HMDB}^{42}$ and $\mathrm{BMRB}^{43}$. The metabolites 3-hydroxybutyrate, isoleucine as well as the 3-methyl-2-oxovalerate that presented low signal to noise ratio were further elucidated by spiking the reference pool sample and performing 2D gHMBCad NMR experiment (Supplementary Figs. S9, S10). To a step further we utilized two specialized software, the online platform Bayesil ${ }^{44}$ and ASICS $^{45}$. Each software proposed a number of metabolites for every sample and provided quantitative results (Supplementary Datasets 1 and 2).

Statistical analysis and identification of discriminant metabolites. Multivariate statistical analysis was employed to aligned spectra after Pareto scaling, using SIMCA software (v. 14.0, Umetrics, Umea, Sweden). At first, PCA was applied to provide a general insight (trends, clusters, outliers) of samples. OPLS-DA was applied next, to generate classification models. Model performance assessed through the $\mathrm{R}^{2} \mathrm{Y}$ (goodness of fit) and $\mathrm{Q}^{2}$ (goodness of prediction) values. Validation of discrimination was made through response permutation testing (999 permutations), analysis of variance (CV-ANOVA) and extraction of ROC curves.

Color coded loadings plots (s-line) attributed certain metabolites responsible for the discrimination pattern. MetaboAnalyst $4.0^{46}$ was applied next to find significant metabolites discriminating umbilical cord blood and maternal groups. Biomarker analysis between IUGR-AGA pairs revealed candidate biomarkers (a cut off value of 0.75 for AUROC was selected) and used for enrichment analysis to provide altered metabolic pathways.

\section{Data availability}

Restrictions apply to the availability of raw data, since were used under license for the current study. Data are however available from the corresponding authors upon justified request.

Received: 16 April 2020; Accepted: 26 March 2021

Published online: 09 April 2021

\section{References}

1. Baschat, A. A. et al. Predictors of neonatal outcome in early-onset placental dysfunction. Obstet. Gynecol. 109, 253-261 (2007).

2. Henriksen, T. Foetal nutrition, foetal growth restriction and health later in life. Acta Paediatr. Suppl. 88, 4-8 (1999).

3. Pankiewicz, K. \& Maciejewski, T. Perinatal mortality and morbidity of growth restricted fetuses and newborns (own experience)first report. Dev. Period Med. 21, 29-34 (2017).

4. Figueroa-Diesel, H., Hernandez-Andrade, E., Acosta-Rojas, R., Cabero, L. \& Gratacos, E. Doppler changes in the main fetal brain arteries at different stages of hemodynamic adaptation in severe intrauterine growth restriction. Ultrasound Obstet. Gynecol. 30, 297-302 (2007).

5. Biri, A. et al. Role of oxidative stress in intrauterine growth restriction. Gynecol. Obstet. Investig. 64, 187-192 (2007).

6. Briana, D. D. \& Malamitsi-Puchner, A. Intrauterine growth restriction and adult disease: The role of adipocytokines. Eur. J. Endocrinol. 160, 337-347 (2009).

7. Devaskar, S. U. \& Chu, A. Intrauterine growth restriction: Hungry for an answer. Physiology 31, 131-146 (2016).

8. Bamfo, J. E. \& Odibo, A. O. Diagnosis and management of fetal growth restriction. J. Pregnancy 2011, 640715. https://doi.org/ $10.1155 / 2011 / 640715(2011)$.

9. Hendrix, N. \& Berghella, V. Non-placental causes of intrauterine growth restriction. Semin. Perinatol. 32, 161-165 (2008).

10. Reynolds, L. P. et al. Evidence for altered placental blood flow and vascularity in compromised pregnancies. J. Physiol. 572, 51-58 (2006).

11. Mandruzzato, G. et al. Intrauterine restriction (IUGR). J. Perinat. Med. 36, 277-281 (2008).

12. Sharma, D., Shastri, S. \& Sharma, P. Intrauterine growth restriction: Antenatal and postnatal aspects. Clin. Med. Insights Pediatr. 10, 67-83 (2016)

13. Alexander, G. R. et al. US birth weight/gestational age-specific neonatal mortality: 1995-1997 rates for whites, hispanics, and blacks. Pediatrics 111, e61-66 (2003).

14. American College of Obstetricians and Gynecologists. ACOG practice bulletin no. 134: Fetal growth restriction. Obstet. Gynecol. 121, 1122-1133 (2013).

15. Lin, G. et al. Metabolomic analysis reveals differences in umbilical vein plasma metabolites between normal and growth-restricted fetal pigs during late gestation. J. Nutr. 142, 990-998 (2012).

16. Favretto, D. et al. Cord blood metabolomic profiling in intrauterine growth restriction. Anal. Bioanal. Chem. 402, 1109-1121 (2012).

17. Conde-Agudelo, A., Papageorghiou, A. T., Kennedy, S. H. \& Villar, J. Novel biomarkers for predicting intrauterine growth restriction: A systematic review and meta-analysis. BJOG Int. J. Obstet. Gynaecol. 120, 681-694 (2013).

18. Dessì, A. \& Fanos, V. Myoinositol: A new marker of intrauterine growth restriction?. J. Obstet. Gynaecol. 33, 776-780 (2013).

19. Priante, E. et al. Intrauterine growth restriction: New insight from the metabolomic approach. Metabolites 9, 267 (2019).

20. Aravidou, E. et al. Protein expression in the brain of rat offspring in relation to prenatal caloric restriction. J. Matern. Fetal. Neonatal. Med. 29, 2707-2714 (2016). 
21. Abd El-Wahed, M. A., El-Farghali, O. G., ElAbd, H. S. A., El-Desouky, E. D. \& Hassan, S. M. Metabolic derangements in IUGR neonates detected at birth using UPLC-MS. Egypt. J. Med. Hum. Genet. 18, 281-287 (2017).

22. Nelson, D. L., \& Cox, M. M. Amino acid oxidation and the production of urea. In Lehninger Principles of Biochemistry 656-689 (Freeman, 2005).

23. Xu, Y., Williams, S. J., O'Brien, D. \& Davidge, S. T. Hypoxia or nutrient restriction during pregnancy in rats leads to progressive cardiac remodeling and impairs postischemic recovery in adult male offspring. FASEB J. 20, 1251-1253 (2006).

24. Newgard, C. B. et al. A branched-chain amino acid-related metabolic signature that differentiates obese and lean humans and contributes to insulin resistance. Cell Metab. 9, 311-326 (2009).

25. Wang, T. J. et al. Metabolite profiles and the risk of developing diabetes. Nat. Med. 17, 448-453 (2011).

26. Menni, C. et al. Biomarkers for type 2 diabetes and impaired fasting glucose using a nontargeted metabolomics approach. Diabetes 62, 4270-4276 (2013).

27. Dessì, A., Marincola, F. C. \& Fanos, V. Metabolomics and the great obstetrical syndromes-GDM, PET, and IUGR. Best Pract. Res. Clin. Obstet. Gynaecol. 29, 156-164 (2015).

28. Human Metabolome Database: Showing metabocard for 3-Methyl-2-oxovaleric acid (HMDB0000491). http://www.hmdb.ca/ metabolites/HMDB0000491.

29. Liu, J., Chen, X.-X., Li, X.-W., Fu, W. \& Zhang, W.-Q. Metabolomic research on newborn infants with intrauterine growth restriction. Medicine 95, e3564 (2016).

30. Nelson, D. L., \& Cox, M. M. Glycolysis, gluconeogenesis, and the pentose phosphate pathway. In Lehninger Principles of Biochemistry 521-559 (Freeman, 2005).

31. Human Metabolome Database: Showing metabocard for Glycerol (HMDB0000131). http://www.hmdb.ca/metabolites/HMDB0 000131.

32. Guo, X. et al. Glycolysis in the control of blood glucose homeostasis. Acta Pharmaceutica Sinica B 2, 358-367 (2012).

33. Bronisz, A., Ozorowski, M. \& Hagner-Derengowska, M. Pregnancy ketonemia and development of the fetal central nervous system. Int. J. Endocrinol. 2018, 1242901 (2018).

34. Powell, K. L. et al. Utility of metabolic profiling of serum in the diagnosis of pregnancy complications. Placenta 66, 65-73 (2018).

35. Cosmi, E. et al. Selective intrauterine growth restriction in monochorionic twin pregnancies: Markers of endothelial damage and metabolomic profile. Twin Res. Hum. Genet. 16, 816-826 (2013).

36. Paolini, C. L. et al. Placental transport of leucine, phenylalanine, glycine, and proline in intrauterine growth-restricted pregnancies. J. Clin. Endocrinol. Metab. 86, 5427-5432 (2001).

37. Sanz-Cortés, M. et al. Metabolomic profile of umbilical cord blood plasma from early and late intrauterine growth restricted (IUGR) neonates with and without signs of brain vasodilation. PLOS ONE 8, e80121 (2013).

38. Economides, D. L., Nicolaides, K. H., Gahl, W. A., Bernardini, I. \& Evans, M. I. Plasma amino acids in appropriate- and small-forgestational-age fetuses. Am. J. Obstet. Gynecol. 161, 1219-1227 (1989).

39. Cetin, I. et al. Maternal concentrations and fetal-maternal concentration differences of plasma amino acids in normal and intrauterine growth-restricted pregnancies. Am. J. Obstet. Gynecol. 174, 1575-1583 (1996).

40. Nagana Gowda, G. A. \& Raftery, D. Quantitating metabolites in protein precipitated serum using NMR spectroscopy. Anal. Chem. 86, 5433-5440 (2014).

41. Filntisi, A. et al. Automated metabolite identification from biological fluid 1H NMR spectra. Metabolomics 13, 146 (2017).

42. Wishart, D. S. et al. HMDB 4.0: The human metabolome database for 2018. Nucleic Acids Res. 46, D608-D617 (2018).

43. Ulrich, E. L. et al. BioMagResBank. Nucleic Acids Res 36, D402-D408 (2008).

44. Ravanbakhsh, S. et al. Accurate, fully-automated NMR spectral profiling for metabolomics. PLoS ONE 10, e0124219 (2015).

45. Tardivel, P. J. C. et al. ASICS: An automatic method for identification and quantification of metabolites in complex 1D 1H NMR spectra. Metabolomics 13, 109 (2017).

46. Chong, J. et al. MetaboAnalyst 4.0: Towards more transparent and integrative metabolomics analysis. Nucleic Acids Res. 46, W486-W494 (2018).

\section{Acknowledgements}

The research work was supported by the Hellenic Foundation for Research and Innovation (HFRI) and the General Secretariat for Research and Technology (GSRT), under the HFRI PhD Fellowship grant (GA. no. 109).

\section{Author contributions}

Conceptualization: T.B., N.I., P.Z. Sample Collection: T.B., Z.I., R.S, N.I. Experimental procedure and NMR analysis: G.M, P.Z. Interpretation of data and statistical analysis: G.M., C.F., P.Z., T.B, Original draft of the manuscript: G.M. Manuscript revision: G.M, C.F, P.Z, All authors reviewed the initial and the revised versions of the manuscript. Resources: T.B., Z.I, R.S, T.X, P.Z. Supervision: N.I, P.Z.

\section{Competing interests}

The authors declare no competing interests.

\section{Additional information}

Supplementary Information The online version contains supplementary material available at https://doi.org/ 10.1038/s41598-021-87323-7.

Correspondence and requests for materials should be addressed to N.I. or P.Z.

Reprints and permissions information is available at www.nature.com/reprints.

Publisher's note Springer Nature remains neutral with regard to jurisdictional claims in published maps and institutional affiliations. 
(c) (i) Open Access This article is licensed under a Creative Commons Attribution 4.0 International cc) License, which permits use, sharing, adaptation, distribution and reproduction in any medium or format, as long as you give appropriate credit to the original author(s) and the source, provide a link to the Creative Commons licence, and indicate if changes were made. The images or other third party material in this article are included in the article's Creative Commons licence, unless indicated otherwise in a credit line to the material. If material is not included in the article's Creative Commons licence and your intended use is not permitted by statutory regulation or exceeds the permitted use, you will need to obtain permission directly from the copyright holder. To view a copy of this licence, visit http://creativecommons.org/licenses/by/4.0/.

(C) The Author(s) 2021 